\title{
A novel three-stage distance-based consensus ranking method
}

\author{
Nazila Aghayi ${ }^{1} \cdot$ Madjid Tavana ${ }^{2,3}$
}

Received: 27 December 2017 / Accepted: 2 April 2018/Published online: 14 May 2018

(C) The Author(s) 2018

\begin{abstract}
In this study, we propose a three-stage weighted sum method for identifying the group ranks of alternatives. In the first stage, a rank matrix, similar to the cross-efficiency matrix, is obtained by computing the individual rank position of each alternative based on importance weights. In the second stage, a secondary goal is defined to limit the vector of weights since the vector of weights obtained in the first stage is not unique. Finally, in the third stage, the group rank position of alternatives is obtained based on a distance of individual rank positions. The third stage determines a consensus solution for the group so that the ranks obtained have a minimum distance from the ranks acquired by each alternative in the previous stage. A numerical example is presented to demonstrate the applicability and exhibit the efficacy of the proposed method and algorithms.
\end{abstract}

Keywords Data envelopment analysis · Multi-criteria decision making · Individual rank · Group rank · Cross-evaluation · Voting

\section{Introduction}

Obtaining a group ranking or a winning candidate from individuals' preferences on a set of alternatives is an important group decision problem with social choice and voting system implications. In a voting system, each voter ranks the alternatives based on his/her preference, so that each alternative may receive different votes in different ranking places. Assume that each voter selects $k$ out of $n$ alternatives provided $k \leq n$ and ranks them from the most to the least preferred. Using the scoring rule, a wellknown ranking system, the total score of each candidate is

Madjid Tavana

tavana@1asalle.edu

http://tavana.us/

Nazila Aghayi

nazila.aghayi@gmail.com

1 Department of Mathematics, Ardabil Branch, Islamic Azad University, Ardabil, Iran

2 Business Systems and Analytics Department, Lindback Distinguished Chair of Information Systems and Decision Sciences, La Salle University, Philadelphia, PA 19141, USA

3 Business Information Systems Department, Faculty of Business Administration and Economics, University of Paderborn, 33098 Paderborn, Germany the weighted sum of votes he or she receives in different place, where the value 1 is assigned to the most important alternative and $\mathrm{n}$ to the least important. Determining the weights used for the different places is clearly an important issue.

Cook and Kress (1990) proposed the data envelopment analysis (DEA) technique to obtain the rank order of alternatives which evaluates each alternative with the most favourable scoring vector. These authors considered the number of votes received at a rank position as an output and used the model with an input equal to unity (Hashimoto 1996) and an additional "assurance region" constraint (Thompson et al. 1986; Hashimoto and Ishikawa 1993). Green et al. (1996) improved this procedure and presented a discrimination method using a cross-efficiency concept, i.e., alternatives take the voters' preference as desired for themselves compared to the other alternatives.

Liamazares and Pena (2009) showed some drawbacks associated with the method presented by Cook and Kress (1990) and introduced methods which recognize the preference between the efficient alternatives. Hashimoto (1997) proposed an AR/exclusion model based upon the concept of super-efficiency presented by Andersen and Petersen (1993). Although Green et al. (1996) proposed a rank order for the alternatives, they did not consider the possibility of assigning a weight of 0 for a given rank or the difference 
between two given ranks to be 0 . Noguchi et al. (2002) presented a strong ordering to alternatives in which weights are obtained using the feasible solution region of the constraint set in LP. Obata and Ishii (2003) introduced a method to discriminate efficient alternatives using ranked voting data without considering information about inefficient alternatives. As such, the rank order presented using this method is independent of inefficient alternatives. Foroughi and Tamiz (2005) extended the model presented by Obata and Ishii (2003) in order to obtain the rank order for both efficient and inefficient alternatives. The extended model contains fewer constraints compared with the model introduced by Obata and Ishii (2003). Discriminating efficient alternatives by considering their least relative total scores was presented by Wang and Chin (2007). Wang et al. (2008) proposed a method to rank multiple efficient alternatives by comparing the least relative total scores for each efficient alternative with the best and the least relative total scores measured in the same range.

Tavana et al. (2007) proposed a new hybrid distancebased ideal-seeking consensus ranking model. Their proposed hybrid model combines parts of the two commonly used consensus ranking techniques of Beck and Lin (1983) and Cook and Kress (1985) into an intuitive and computationally simple model. Tavana et al. (2008) proposed a new weighted sum ordinal Consensus ranking method with the weights derived from a Sigmoid function. They ran Monte Carlo simulation to compare the similarity of the consensus rankings generated by our method with the bestknown method of Borda-Kendall and two other commonly used techniques. They showed although consensus rankings generated by different algorithms are similar, differences in rankings among the algorithms were of sufficient magnitude that they often cannot be viewed as interchangeable from a practical perspective.

Zerafat Angiz et al. (2009) proposed a multi-objective linear programming DEA based model to select the best alternative in a group decision-making environment Contreras (2010) presented a distance-based consensus model with flexible choice of rank-position weights in which preference aggregation is obtained by a ranking of alternatives. To do so, a mixed integer linear programming model was constructed providing the preference of alternatives by the vector of weights that minimizes the disagreement across decision makers. In addition to this model, Contreras (2011) proposed another method that ranks the alternatives in two stages. First stage is based on the cross-evaluation methodology in which (1) the rank of alternatives is computed in their best condition and (2) the individual rank of each alternative is obtained. In the second stage, the group rank of alternatives with common weights is obtained. Hosseinzadeh Lotfi et al. (2013) worked on a three-stage process to rank alternatives. Based on this model, in the first stage the alternatives are evaluated in their best condition with the DEA model. Since the optimal weights obtained in the first stage are non-unique, a second stage is introduced in order to limit the vector of weights. In the third stage, the group rank position is determined based upon the minimum distance by the mean rank obtained in the second stage. To discriminate between efficient alternatives, Soltanifar and Hosseinzadeh Lotfi (2011) used the voting analytic hierarchy process method (VAHP).

The DEA evaluation cannot derive a unique optimal weight vector for the alternatives. So, the problem mentioned above makes the cross-evaluation important. In DEA, to deal with this problem, Sexton et al. (1986) initially proposed a secondary goal and then Doyle and Green (1994) and Doyle and Green (1995) suggested the most widely used secondary goals (i.e., aggressive and benevolent evaluation). Lianga et al. (2008) extended the model introduced by Doyle and Green (1994) as utilizing an alternative secondary goal. Contreras (2012) optimized the rank positions of alternatives as a secondary goal in cross-evaluation so that the alternatives could assume tie ranks.

Ziari, and Raissi (2016) ranked extreme efficient DMUs that solve the infeasibility and unboundedness problem of other methods. Their approach minimized the distance between under evaluation and virtual DMUs. Hafezalkotob and Hafezalkotob (2017) used the interval target-based norm and also considered the concept of degree of preference of interval numbers in order to rank these numbers. Ziari (2016) developed an alternative method to convert the nonlinear model of ranking the DMUs using the $\mathrm{L}_{1}$ norm that has been introduced by Jahanshahloo et al. (2004). Ding and Kamaruddin (2015) compared both crisp TOPSIS and fuzzy TOPSIS from group decision making based on the distance concept. Tohidi and Razavyan (2012) introduced the recession direction for a multi-objective integer linear programming problem.

Gong et al. (2018) used DEA models to evaluate preference in voting system with abstentions. Ebrahimnejad et al. (2016) applied the DEA method to rank efficient alternatives and used simulation to analyse the rankings and synthesize them into one group ranking. Gong et al. (2015) proposed the models to evaluate the consensus rank. Their model is based on minimum cost and maximum return. They used interval preferences for individual ranks. Liu et al. (2017) obtained consensus in group decision making based on an interval-valued trust decision-making space. Wu et al. (2018) introduced a consensus model for social network group decision-making problems. Zhang et al. (2017) presented the consensus models based on minimum cost by random opinions. Also, they discussed 
sensitivity analysis for different opinions and distributions of cases.

We propose a three-stage method for the ranking of the alternatives in a voting system in a way to minimize the distance between the individual and group ranks. In the first stage, the optimistic rank of each alternative is determined. Each alternative is evaluated not only with its optimal weights but also with the remaining alternatives' weights, implying that the vector of optimal weights presented is not unique. Although the given model has unique objective values, the vector obtained does not necessarily have a unique value. Thus, depending on which vector is selected, the rank position of other alternatives can alter. Consequently, a secondary goal is introduced to limit the optimal weight vector in the second stage. In the third stage, the ranking of alternatives is computed by common weights in a way that the group ranks have minimum distance from each individual rank by different norms. The proposed model assigns integer ranks to the alternatives. It is important to mention that this is a multi-criteria decisionmaking model which we solve using mixed integer programming.

The remainder of this paper is organized as follows. In "Proposed method" section introduces the proposed threestage ranking method. In "Numerical example" section a numerical example is provided to demonstrate the applicability and exhibit the efficacy of the proposed method. In "Conclusion and future research directions" section highlights our conclusions and future research directions.

\section{Proposed method}

\section{Individual rank position}

One of the most important aspects of decision making is to obtain a group ranking using the individual ranking for a certain set of alternatives. Assume that $n$ alternatives $\left\{x_{1}, \ldots, x_{n}\right\}$ with $n \geq 3$ have to be assigned to $k(k \leq n)$ places. There are many methods for determining the ranks of alternatives using their weights. For instance, scoring rules compute the score for each alternative based on its rank position in the individual preference and then rank the alternatives by the sum of resulting scores. Assuming that $v_{i j}$ indicates the number of votes that $x_{i}$ receives in the $j$ th rank position and $w_{j}$ represents the weight or score associated with $v_{i j}$, then the aggregate value is defined for the alternative $x_{i}$ as the weighted sum of the votes receives in different places, i.e., $V\left(x_{i}\right)=\sum_{j=1}^{k} w_{j} v_{i j}$. Therefore, the alternatives can be ranked by comparing their aggregate values. Moreover, the voting system can be used to rank the alternatives. The value of 1 and $n$ are given to the most and least important (preferences) alternatives, respectively. In addition, the ranks are distinct. Hence, the rank vector of alternatives is from 1 to $n$ defining a linear order for the alternatives. Hosseinzadeh Lotfi et al. (2013) suggested a three-stage method in order to rank alternatives. They employed a model for ranking alternatives in the first stage by considering the best rank position of the alternative under evaluation. To do this, a mixed integer linear model is solved as follows (see Model 1):

$\min r_{o}^{o}$

s.t. $\quad \sum_{j=1}^{k} w_{j}^{o} v_{i j}-\sum_{j=1}^{k} w_{j}^{o} v_{h j}+\delta_{i h}^{o} M \geq 0, \quad \forall i, h, i \neq h$,

$\delta_{i h}^{o}+\delta_{h i}^{o}=1, \quad \forall i, h, \quad i \neq h$,

$\delta_{i h}^{o}+\delta_{h l}^{o}+\delta_{l i}^{o} \geq 1, \quad \forall i, h, l, i \neq h \neq l$,

$r_{i}^{o}=1+\sum_{i \neq h} \delta_{i h}^{o}, \quad \forall i$

$w_{j}^{o} \in \phi, \quad \forall j$

$\delta_{i h}^{o} \in\{0,1\}, \quad \forall i, h$,

where $M$ is a large positive number. $w_{j}^{o}$ indicates associated weights of votes in the $j$ th place when alternative $x_{o}$ is evaluated and $v_{i j}$ shows the number of votes that the alternative $x_{i}$ is received in the $j$ th place. Accordingly, $V^{o}\left(x_{i}\right)=\sum_{j=1}^{k} w_{j}^{o} v_{i j}$ states the aggregate value of votes as the optimal weight vector of the alternative under evaluation. Let $V^{o}\left(x_{i}\right) \geq V^{o}\left(x_{h}\right)$ then the rank position of $x_{i}$ is better than $x_{h}$, and in addition, $\delta_{i h}^{o}=0$ represents a better rank position for $x_{i}$ rather than $x_{h}$, where $\delta_{i h}^{o}$ is a binary variable. If $\delta_{i h}^{o}=0$, then constraint (1a) in Model (1) is obtained as follows:

$$
\begin{aligned}
& \sum_{j=1}^{k} w_{j}^{o} v_{i j}-\sum_{j=1}^{k} w_{j}^{o} v_{h j}+\delta_{i h}^{o} M \geq 0 \\
& \Rightarrow \sum_{j=1}^{k} w_{j}^{o} v_{i j} \geq \sum_{j=1}^{k} w_{j}^{o} v_{h j} \Rightarrow V^{o}\left(x_{i}\right) \geq V^{o}\left(x_{h}\right)
\end{aligned}
$$

Therefore, the rank position of $x_{i}$ is better than that of $x_{h}$. The constraints (1a) are redundant if $\delta_{i h}^{o}=1$. It is presumed that alternatives are ranked from 1 to $n$, implying that the alternatives do not have equal ranks which makes constraints (1b) and (1c) necessary. Constraints (1b) demonstrate that $V^{o}\left(x_{i}\right)$ and $V^{o}\left(x_{h}\right)$ are comparable, i.e., either the rank position of alternative $x_{i}$ is better than $x_{h}$ or the rank position of alternative $x_{h}$ is better than $x_{i}$. Constraints (1d) indicate the number of times that $x_{1}$ is worse than $x_{2}$ and the Term $R^{o}=\left(r_{1}^{o}, \ldots, r_{n}^{o}\right)$ explains the preference vector obtained in the evaluation of alternative $x_{o}$. It should be 
noted that the weights are computed assuming that the alternatives are located in the best rank position. In addition, the rank of each alternative is integer-valued.

In order to establish a consensus between several DMs about selecting weights, a feasible set $\phi$ is defined for the weights. This includes minimum information about the discrimination between the components of the weight vector $w^{o}$. Therefore, the set $\phi$ should be specified as $\phi=\left\{w^{o} \in R^{k} \mid w_{1}^{o} \geq w_{2}^{o} \geq \cdots \geq w_{k}^{o} \geq 0, \sum_{j=1}^{k} w_{j}^{o}=1\right\}$. If the set $\phi$ contains only one vector, the rank position of alternatives will be then given based on their aggregate values. As previously mentioned, the set $\phi$ includes the preference vectors between weights, so that an order is determined for alternatives using $V^{o}\left(x_{i}\right)$ based on each vector $w^{o}$. As a result, a criterion is proposed to characterize the set $\phi$ in the evaluation of alternatives. For example, the set $\phi$ can be defined as $\phi=\left\{w^{o} \in R^{k} \mid w_{1}^{o}-\right.$ $\left.w_{2}^{o} \geq w_{2}^{o}-w_{3}^{o}, w_{2}^{o}-w_{3}^{o} \geq w_{3}^{o}-w_{4}^{o}, \ldots\right\}$ which shows the discrimination between the components of the weights vector (for example, see Contreras et al. 2005; Cook and Kress 1996).

\section{Secondary goal}

In general, Model (1) is run $n$ times and introduces a rank vector in each run. We say a rank matrix $R$ of order $n$ is generated in which the oth column is given by $R^{o}=\left(r_{1}^{o}, \ldots, r_{n}^{o}\right)$. Since the ranks presented can be changed due to the non-uniqueness of the optimal weights obtained from Model (1), the elements of the rank matrix $R$, excluding the diagonal elements, can then vary depending on which vector is used. In fact, there exists a problem similar to the problem of the cross-evaluation using DEA. The vector of weights derived from the evaluation using DEA is not unique. As a result, $r_{i}^{o},(i \neq o)$ may not take a unique value. To solve this problem, the DEA applies a secondary goal. In here, we suggest the following secondary goal in order to limit the optimal solutions obtained by the evaluation of alternatives in the first stage:

$$
\begin{aligned}
& \min \quad \sum_{o=1}^{n} r_{o o}+\varepsilon \sum_{o=1}^{n} \sum_{i<o} \sum_{j=1}^{k}\left|w_{j o}-w_{j i}\right| \\
& \text { s.t. } \quad \sum_{j=1}^{k} w_{j o} v_{i j}-\sum_{j=1}^{k} w_{j o} v_{h j}+\delta_{i h o} M \geq 0, \quad \forall o, i, h, i \neq h, \\
& \delta_{\text {iho }}+\delta_{\text {hio }}=1, \quad \forall o, i, h, i \neq h, \\
& \delta_{\text {iho }}+\delta_{\text {hlo }}+\delta_{\text {lio }} \geq 1, \quad \forall o, i, h, l, i \neq h \neq l,
\end{aligned}
$$

$r_{i o}=1+\sum_{i \neq h} \delta_{i h o}, \quad \forall o, i$,

$w_{j o} \in \phi, \quad \forall o, j$,

$\delta_{\text {iho }} \in\{0,1\}, \quad \forall o, i, h$.

Using Model (1), each alternative is evaluated provided the alternative under evaluation is estimated in the optimistic case. Therefore, the weights obtained from each run of Model (1) can vary significantly from each other. Hence, in Model (2), Model (1) is written in an integrated form in order to select close-up weights for each alternative where each alternative is evaluated independently. Moreover, using Model 2, the weights can be compared with each other. As mentioned earlier, $M$ is a large positive number. The term $w_{j i}$ signifies the weights assigned to the $j$ th place in the evaluation of alternative $x_{i}$. The term $v_{i j}$ gives the number of votes that the alternative $x_{i}$ receives in the $j$ th place. Furthermore, $\delta_{i h o}$ is the same as the binary variable $\delta_{i h}^{o}$. The term $r_{i o}$ indicates the element of the rank matrix $R$ that lies in the $i$ th row and the oth column. Model (2) is a two-objective model which minimizes the sum of the ranks and the difference of the weights obtained using Model (1) as the first and second objectives, respectively. The purpose of the model is to choose the alternative under evaluation which has the best rank position. The coefficient $\varepsilon$ is suggested as the difference of the weights where $\varepsilon$ is a nonArchimedean number. Moreover, Model (2) is a nonlinear model, meaning that it can be converted to a mixed integer linear model via changes of the variables $w_{o j}-w_{i j}=d_{o i j}^{w}$. Therefore, the constraints $d_{o i j}^{w} \geq w_{o j}-w_{i j} \quad$ and $d_{o i j}^{w} \geq w_{i j}-w_{o j}$, for all $i, j, i<o$, are added to the model. In order to obtain the rank matrix $R$ that includes the individual ranks, the following model is solved:

$$
\begin{array}{ll}
\min & \sum_{o=1}^{n} r_{o o}+\varepsilon \sum_{o=1}^{n} \sum_{i<o} \sum_{j=1}^{k} d_{i j o}^{w} \\
\text { s.t. } & \sum_{j=1}^{k} w_{j o} v_{i j}-\sum_{j=1}^{k} w_{j o} v_{h j}+\delta_{i h o} M \geq 0, \quad \forall o, i, h, i \neq h,
\end{array}
$$

$\delta_{\text {iho }}+\delta_{\text {hio }}=1, \quad \forall o, i, h, i \neq h$,

$\delta_{\text {iho }}+\delta_{\text {hlo }}+\delta_{\text {lio }} \geq 1, \quad \forall o, i, h, l, i \neq h \neq l$,

$r_{i o}=1+\sum_{i \neq h} \delta_{i h o}, \quad \forall o, i$,

$d_{i j o}^{w} \geq w_{j o}-w_{j i}, \quad \forall o, i, j, i<o$,

$d_{i j o}^{w} \geq w_{j i}-w_{j o}, \quad \forall o, i, j, i<o$,

$w_{j o} \in \phi, \quad \forall o, j$, 
$\delta_{\text {iho }} \in\{0,1\}, \quad \forall o, i, h$,

$d_{i j o}^{w} \geq 0, \quad \forall o, i, j$.

\section{Group rank position}

As previously mentioned, $R^{o}=\left(r_{1 o}, \ldots, r_{n o}\right)$ denotes the rank vector presented in the evaluation of alternative $x_{o}$. In order to obtain a group rank $R^{\mathrm{G}}=\left(r_{1}^{\mathrm{G}}, \ldots, r_{n}^{\mathrm{G}}\right)$ considering $R^{o}=\left(r_{10}, \ldots, r_{n o}\right)$ obtained from Model (3), in the third stage, a multi-objective mathematical model is provided. This model selects a common vector of weights from the set $\phi$ that minimizes the disagreement between all individual and group ranks. Hence, the distance function $d^{p}\left(R^{\mathrm{G}}, R\right)=\sum_{h=1}^{n} d^{p}\left(R^{\mathrm{G}}, R^{h}\right)=\sum_{h=1}^{n}\left(\sum_{i=1}^{n}\left|r_{i}^{\mathrm{G}}-r_{i h}\right|^{p}\right)^{\frac{1}{p}}$, in which $r_{i}^{\mathrm{G}}$ is the $i$ th component of the group rank and $r_{i h}$ which measures the rank of $x_{i}$ in the evaluation of $x_{h}$, is used to minimize the distance between the individual and group ranks. The metrics $l^{1}, l^{2}$, and $l^{\infty}$ are defined for $p=1, p=2$, and $p=\infty$, respectively, where:

$$
\begin{aligned}
d^{1}\left(R^{\mathrm{G}}, R\right) & =\sum_{h=1}^{n} \sum_{i=1}^{n}\left|r_{i}^{\mathrm{G}}-r_{i h}\right|, d^{2}\left(R^{\mathrm{G}}, R\right) \\
& =\sum_{h=1}^{n}\left(\sum_{i=1}^{n}\left(r_{i}^{\mathrm{G}}-r_{i h}\right)^{2}\right)^{\frac{1}{2}} \text { and } d^{\infty}\left(R^{\mathrm{G}}, R\right) \\
& =\max _{i, h}\left|r_{i}^{\mathrm{G}}-r_{i h}\right| .
\end{aligned}
$$

Considering these, the following model is introduced:

$$
\begin{array}{ll}
\min & d^{1}\left(R^{\mathrm{G}}, R\right) \\
\min \quad & d^{2}\left(R^{\mathrm{G}}, R\right) \\
\min \quad & d^{\infty}\left(R^{\mathrm{G}}, R\right) \\
\text { s.t. } \quad \sum_{j=1}^{k} w_{j} v_{i j}-\sum_{j=1}^{k} w_{j} v_{h j}+\delta_{i h} M \geq 0, \quad \forall i, h, i \neq h, & \\
\delta_{i h}+\delta_{h i}=1, \quad \forall i, h, i \neq h, \\
\delta_{i h}+\delta_{h l}+\delta_{l i} \geq 1, \quad \forall i, h, l, i \neq h \neq l, \\
r_{i}^{\mathrm{G}}=1+\sum_{i \neq h} \delta_{i h}, \quad \forall i, \\
w_{j} \in \phi, \quad \forall j, \\
\delta_{i h} \in\{0,1\}, \quad \forall i, h .
\end{array}
$$

It should be noted that the group rank estimated by the metrics $l^{1}$ and $l^{2}$ is the consensus rank that can be interpreted as the median and mean distance statistics, respectively. Indeed, the median and mean ranks are obtained based on the median and mean of the row ranks of each alternative in the rank matrix $R$ produced by Model (3). The rank group is computed in a way that has a minimum distance from the group rank of each alternative using its corresponding median and mean ranks, respectively. It is important to mention that the outlier ranks have a less preference by using the metric $l^{1}$ and the metric $l^{2}$ considers the effect of a rank with different weights for each alternative. The metric $l^{\infty}$ also minimizes the maximum distance between the individual and group ranks. Note that it avoids creating the maximum distance between two ranks for one place. It is obvious that Model (4) is a nonlinear model. In order to write a mixed integer linear model, we use the following relationships:

- Nonnegative variables $d_{i h}^{r_{1}}$ are defined to obtain the linear form of $d^{1}\left(R^{\mathrm{G}}, R\right)$ provided that $d_{i h}^{r_{1}}=r_{i}^{\mathrm{G}}-r_{i h}, \forall i, h . \quad$ Therefore, the constraints $d_{i h}^{r_{1}} \geq r_{i}^{\mathrm{G}}-r_{i h}$ and $d_{i h}^{r_{1}} \geq r_{i h}-r_{i}^{\mathrm{G}}, \forall i, h$ are added to the linear model.

- In order to have a linear form for $d^{2}\left(R^{\mathrm{G}}, R\right)$, the derivative of the distance function with respect to $r_{i}^{\mathrm{G}}$ can be calculated because of the convexity property of $d^{2}\left(R^{\mathrm{G}}, R\right)$. That is;

$$
\begin{aligned}
\frac{d\left(d^{2}\left(R^{\mathrm{G}}, R\right)\right)}{d\left(r_{i}^{\mathrm{G}}\right)} & =\sum_{h=1}^{n}\left(r_{i}^{\mathrm{G}}-r_{i h}\right)=\left(n r_{i}^{\mathrm{G}}-\sum_{h=1}^{n} r_{i h}\right) \\
& =n\left(r_{i}^{\mathrm{G}}-\frac{1}{n} \sum_{h=1}^{n} r_{i h}\right) .
\end{aligned}
$$

The minimum value of $d^{2}\left(R^{\mathrm{G}}, R\right)$ is obtained by setting the derivative equal to 0 . Hence, we have

$$
\begin{aligned}
\min _{i} d^{2}\left(R^{\mathrm{G}}, R\right) & =\min \sum_{i=1}^{n}\left|\frac{d\left(d^{2}\left(R^{\mathrm{G}}, R\right)\right)}{d\left(r_{i}^{\mathrm{G}}\right)}-0\right| \\
& =\min \sum_{i=1}^{n}\left|n\left(r_{i}^{\mathrm{G}}-\frac{1}{n} \sum_{h=1}^{n} r_{i h}\right)\right| \\
& =\min \sum_{i=1}^{n}\left|r_{i}^{\mathrm{G}}-\bar{r}_{i}\right|
\end{aligned}
$$

where $\bar{r}_{i}=\frac{1}{n} \sum_{h=1}^{n} r_{i h}$ is interpreted as the mean value of the $i$ th row ranks of the alternative $x_{i}$. Thus, the nonnegative variables $d_{i}^{r_{2}}=r_{i}^{\mathrm{G}}-\bar{r}_{i}, \forall i$ are defined and the constraints $d_{i}^{r_{2}} \geq r_{i}^{\mathrm{G}}-\bar{r}_{i}$ and $d_{i}^{r_{2}} \geq \bar{r}_{i}-r_{i}^{\mathrm{G}}, \forall i$ are added to the linear form of the model.

- The term $d^{r_{\infty}}=\max _{i, h}\left|r_{i}^{\mathrm{G}}-r_{i h}\right|$ is considered to derive the linear form of $d^{\infty}\left(R^{\mathrm{G}}, R\right)$ subject to $d^{r_{\infty}} \geq d_{i h}^{r_{1}}$.

Considering the above, the linear form of Model (4) is as follows: 


$$
\begin{array}{ll}
\min & \sum_{i=1}^{n} \sum_{h=1}^{n} d_{i h}^{r_{1}} \\
\min & \sum_{i=1}^{n} d_{i}^{r_{2}} \\
\min \quad d^{r_{\infty}} & \\
\text { s.t. } \quad \sum_{j=1}^{k} w_{j} v_{i j}-\sum_{j=1}^{k} w_{j} v_{h j}+\delta_{i h} M \geq 0, \quad \forall i, h, i \neq h, \\
\delta_{i h}+\delta_{h i}=1, \quad \forall i, h, i \neq h, \\
\delta_{i h}+\delta_{h l}+\delta_{l i} \geq 1, \quad \forall i, h, l, i \neq h \neq l,
\end{array}
$$

group rank close to the median value, $v_{1}$ will take a bigger value than $v_{2}$ and $v_{3}$. The weight $v_{2}$ will have the most importance if the value of $v_{2}$ is more than other weights.

In fact, if the DM wants the group rank close to the median value, then he can use the metric 11. In this case, the outlier ranks have less preference. If the DM wants the group rank close to the mean value, then he can use the metric 12. In this case, the effect of a rank with different weights is presented. $l^{\infty}$ minimizes the maximum distance between the individual and group rank. Therefore, the DM can compute the group rank position of alternatives by different norms. The pseudocode of our algorithm is presented here:

Start;

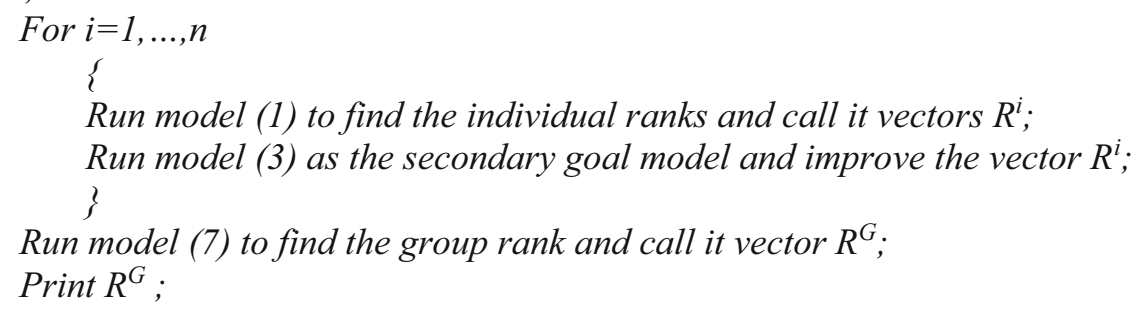

$r_{i}^{G}=1+\sum_{i \neq h} \delta_{i h}, \quad \forall i$,

$d_{i h}^{r_{1}} \geq r_{i h}-r_{i}^{\mathrm{G}}, \quad \forall i, h$,

$d_{i h}^{r_{1}} \geq r_{i}^{\mathrm{G}}-r_{i h}, \quad \forall i, h$,

$d^{r_{\infty}} \geq d_{i h}^{r_{1}}, \quad \forall i, h$,

$d_{i}^{r_{2}} \geq \frac{1}{n} \sum_{h=1}^{n} r_{i h}-r_{i}^{\mathrm{G}}, \quad \forall i$

$d_{i}^{r_{2}} \geq r_{i}^{\mathrm{G}}-\frac{1}{n} \sum_{h=1}^{h} r_{i h}, \quad \forall i$,

$w_{j} \in \phi, \quad \forall j$,

$\delta_{i h} \in\{0,1\}, \quad \forall i, h$,

$d_{i h}^{r_{1}}, d_{i}^{r_{2}}, d^{r_{\infty}} \geq 0, \quad \forall i, h$.

The above model is a mixed integer multi-objective model which can be solved using many methods. To solve Model (7), the weighted sum method is used so that the used weights are standardized. Therefore, the objective functions of Model (7) can be replaced with one objective, $\min \frac{v_{1}}{n^{2}} \sum_{i=1}^{n} \sum_{h=1}^{n} d_{i h}^{r_{1}}+\frac{v_{2}}{n} \sum_{i=1}^{n} d_{i}^{r_{2}}+v_{3} d^{r_{\infty}}$, to compute the group rank position of the alternatives where $v_{1}, v_{2}$, and $v_{3}$ are the weights assigned to the three objectives by the DMs and are nonnegative values. If the DMs wish the

\section{Numerical example}

In this section, we apply our method to Green et al. (1996) problem where voters are asked to rank seven alternatives. Table 1 shows the number of votes for each alternative.

Let the consensus of DMs on selecting the weights that show the discrimination between the components of the weight vector be given by $\phi=\left\{w_{1} \geq w_{2} \geq 0.01, w_{1}+\right.$ $\left.w_{2}=1\right\}$. The set $\phi$ can be interpreted as the weight of the first position of votes is more than the second position. The lower value for the weight of the second position is 0.01 , and the sum of the both places is equal to one. As previously mentioned, the alternatives are evaluated in their optimistic cases as the best rank position for the alternatives is computed in Stage 1. Since Model (1) may have multiple optimal weights, so the secondary goal is suggested to deal with the above mentioned problem. Hence, Model (3) is run in order to obtain the rank matrix $R$. This minimizes the rank position of the alternative under evaluation as well as the weights difference considered in the first and second position votes. Table 2 shows the rank position of all the alternatives in Stage 2. The weighting vector is presented in Table 3.

In the third stage, the distance between the group rank $R^{\mathrm{G}}$ and each column of the rank matrix is minimized by considering the metrics $l^{1}, l^{2}$, and $l^{\infty}$ where the DM 
Table 1 The votes

\begin{tabular}{lll}
\hline Alternatives & First place & Second place \\
\hline$x_{1}$ & 32 & 10 \\
$x_{2}$ & 28 & 20 \\
$x_{3}$ & 13 & 36 \\
$x_{4}$ & 20 & 27 \\
$x_{5}$ & 27 & 19 \\
$x_{6}$ & 30 & 8 \\
$x_{7}$ & 0 & 30 \\
\hline
\end{tabular}

Table 2 Rank position of alternatives in the second stage

\begin{tabular}{llllllll}
\hline Alternatives & $r_{i}^{1}$ & $r_{i}^{2}$ & $r_{i}^{3}$ & $r_{i}^{4}$ & $r_{i}^{5}$ & $r_{i}^{6}$ & $r_{i}^{7}$ \\
\hline$x_{1}$ & 1 & 2 & 6 & 5 & 3 & 4 & 7 \\
$x_{2}$ & 2 & 1 & 6 & 4 & 3 & 5 & 7 \\
$x_{3}$ & 5 & 2 & 1 & 3 & 4 & 6 & 7 \\
$x_{4}$ & 5 & 1 & 4 & 3 & 2 & 6 & 7 \\
$x_{5}$ & 3 & 1 & 6 & 4 & 2 & 5 & 7 \\
$x_{6}$ & 1 & 3 & 6 & 5 & 4 & 2 & 7 \\
$x_{7}$ & 2 & 1 & 6 & 4 & 3 & 5 & 7 \\
\hline
\end{tabular}

Table 3 The value of weights

\begin{tabular}{lll}
\hline Alternatives & $w_{1}$ & $w_{2}$ \\
\hline$x_{1}$ & 0.714 & 0.286 \\
$x_{2}$ & 0.643 & 0.357 \\
$x_{3}$ & 0.516 & 0.484 \\
$x_{4}$ & 0.578 & 0.422 \\
$x_{5}$ & 0.643 & 0.357 \\
$x_{6}$ & 0.857 & 0.143 \\
$x_{7}$ & 0.643 & 0.357 \\
\hline
\end{tabular}

presents the weights as $v_{1}=0.4, v_{2}=0.4$, and $v_{3}=0.2$. In this stage, the weights are obtained based on the common vector of weights, $\phi$. The resulting group rank is $R^{\mathrm{G}}=(3,1,5,4,2,6,7)$. The weight vector obtained from the optimal solution is $w=(0.622,0.378)$. It should be noted that different values of $v_{1}, v_{2}$, and $v_{3}$ selected by the DMs give a different group rank. In fact, if the DMs wish the group rank to have the least distance with the median values of the rank matrix, he/she can consider $v_{1}$ to be the most important. Similarly, the most preference can be given to $v_{2}$ when the DMs desire to obtain the group rank close to the mean of the rank matrix. $v_{3}$ also can take a higher value than $v_{1}$ and $v_{2}$, when the DMs want to minimize the maximum difference. Thus, the choice of $v_{1}, v_{2}$, and $v_{3}$ reflect the DM's objectives.
We have $w=(0.622,0.378)$ in the numerical example and use the votes in Table 1 . We obtain the following results:

$$
\begin{aligned}
V^{o}\left(x_{1}\right) & =23.684 \\
V^{o}\left(x_{2}\right) & =24.976 \\
V^{o}\left(x_{3}\right) & =21.694 \\
V^{o}\left(x_{4}\right) & =22.646 \\
V^{o}\left(x_{5}\right) & =23.976 \\
V^{o}\left(x_{7}\right) & =11.34 \\
V^{o}\left(x_{6}\right) & =21.684
\end{aligned}
$$

If $V^{o}\left(x_{i}\right) \geq V^{o}\left(x_{h}\right)$, then the rank position of $x_{i}$ is better than that of $x_{h}$. Therefore, $R^{\mathrm{G}}=(3,1,5,4,2,6,7)$.

\section{Conclusion and future research directions}

In this study, we proposed a three-stage method to rank alternatives in the voting system. In the first stage, the rank position of each alternative was computed based on the weight vector of the alternative under evaluation. The model in Stage 1 was run $n$ times to produce a set of rankings for each run. Consequently, a rank matrix of order $n$ was obtained in which the $i$ th column signifies the rank vector when the alternative under evaluation is $x_{i}$. Since the vector of weights obtained in the first stage is not a singleton, the rank position of the alternative under evaluation remains unchanged, but the rank matrix obtained can then vary when the weights change. To deal with this problem, a secondary goal was defined in Stage 2 of the method. The secondary goal aimed at minimizing weights differences in each alternative. In the third stage of the method, the group rank position of alternatives was computed using the CSW for all the alternatives based on a distance of individual rank positions. In fact, the third stage determined a consensus solution for the group so that the ranks obtained have a minimum distance from the ranks acquired by each alternative in the previous stage. The minimum distance can be obtained by the metrics $l^{1}, l^{2}$, and $l^{\infty}$ as selected by the DMs. In this model, all three metrics can be employed, and the DMs can choose one, two, or three of them. The DMs can use the metrics $l^{1}$ and $l^{2}$ when the group rank close to median and mean values are required, respectively. The DMs also can consider $l^{\infty}$ to obtain a group rank as it minimizes the maximum distance between the individual and group ranks. The proposed model is a multi-criteria decision-making model that can be converted to a model with one objective by the weighted sum method.

Acknowledgement Dr. Nazila Aghayi is grateful for the financial support and the sabbatical opportunity provided to her by the Ardabil Branch, Islamic Azad University to attend the Universite Catholique de Louvain and conduct this research. 
Open Access This article is distributed under the terms of the Creative Commons Attribution 4.0 International License (http://creative commons.org/licenses/by/4.0/), which permits unrestricted use, distribution, and reproduction in any medium, provided you give appropriate credit to the original author(s) and the source, provide a link to the Creative Commons license, and indicate if changes were made.

\section{References}

Andersen P, Petersen NC (1993) A procedure for ranking efficient units in data envelopment analysis. Manage Sci 39(10):1261-1294

Beck MP, Lin BW (1983) Some heuristics for the consensus ranking problem. Comput Oper Res 10(1):1-7

Contreras I (2010) A distance-based consensus model with flexible choice of rank-position weights. Group Decis Negot 19:441-456

Contreras I (2011) A DEA-inspired procedure for the aggregation of preferences. Expert Syst Appl 38:564-570

Contreras I (2012) Optimizing the rank position of the DMU as secondary goal in DEA cross-evaluation. Appl Math Model 36(6):2642-2648

Contreras I, Hinojosa MA, Mármol AM (2005) A class of flexible weight indices for ranking alternatives. IMA J Manag Math 16(1):71-85

Cook WD, Kress M (1985) Ordinal ranking with intensity of preference. Manage Sci 31(1):26-32

Cook WD, Kress M (1990) A data envelopment model for aggregating preference rankings. Manage Sci 36:1302-1310

Cook WD, Kress M (1996) An extreme-point approach for obtaining weighted ratings in qualitative multi criteria decision making. Nav Res Logist 43(4):519-531

Ding SH, Kamaruddin S (2015) Assessment of distance-based multiattribute group decision-making methods from a maintenance strategy perspective. J Ind Eng Int 11(1):73-85

Doyle J, Green R (1994) Efficiency and cross-efficiency in DEA: derivations, meanings and uses. J Oper Res Soc 45:567-578

Doyle J, Green RH (1995) Cross-evaluation in DEA: improving discrimination among DMUs. INFOR 33:205-222

Ebrahimnejad A, Tavana M, Santos-Arteaga FJ (2016) An integrated data envelopment analysis and simulation method for group consensus ranking. Math Comput Simul 119:1-17

Foroughi AA, Tamiz M (2005) An effective total ranking model for a ranked voting system. Omega 33:491-496

Gong ZW, Xu XX, Zhang HH, Ozturk UA, Herrera-Viedma E, Xu C (2015) The consensus models with interval preference opinions and their economic interpretation. Omega 55:81-90

Gong Z, Zhang N, Li KW, Martínez L, Zhao W (2018) Consensus decision models for preferential voting with abstentions. Comput Ind Eng 115:670-682

Green RH, Doyle JR, Cook WD (1996) Preference voting and project ranking using DEA and cross-evaluation. Eur J Oper Res 90(3):461-472

Hafezalkotob A, Hafezalkotob A (2017) Interval MULTIMOORA method with target values of attributes based on interval distance and preference degree: biomaterials selection. J Ind Eng Int 13(2):181-198

Hashimoto A (1996) DEA selection system for selection examinations. J Oper Res Soc Jpn 39(4):475-485

Hashimoto A (1997) A ranked voting system using a DEA/AR exclusion model, a note. Eur J Oper Res 97:600-604

Hashimoto H, Ishikawa H (1993) Using DEA to evaluate the state of society as measured by multiple social indicators. Socioecon Plann Sci 27(4):257-268
Hosseinzadeh Lotfi F, Rostamy-Malkhalifeh M, Aghayi N, Gelej Beigi Z, Gholami K (2013) An improved method for ranking alternatives in multiple criteria decision analysis. Appl Math Model 37(1-2):25-33

Jahanshahloo GR, Hosseinzadeh Lotfi F, Shoja N, Tohidi G, Razavian S (2004) Ranking by using L1-norm in data envelopment analysis. Appl Math Comput 153:215-224

Liamazares B, Pena T (2009) Preference aggregation and DEA: an analysis of the methods proposed to discriminate efficient candidates. Eur J Oper Res 197:714-721

Lianga L, Wua J, Cook WD, Zhu J (2008) Alternative secondary goals in DEA cross-efficiency evaluation. Int $\mathrm{J}$ Prod Econ 113:1025-1030

Liu Y, Liang C, Chiclana F, Wu J (2017) A trust induced recommendation mechanism for reaching consensus in group decision making. Knowl-Based Syst 119:221-231

Noguchi H, Ogawa M, Ishii H (2002) The appropriate total ranking method using DEA for multiple categorized purposes. J Comput Appl Math 146:155-166

Obata T, Ishii H (2003) A method for discriminating efficient candidates with ranked voting data. Eur J Oper Res 151:233-237

Sexton TR, Silkman RH, Hogan AJ (1986) Data envelopment analysis: Critique and extensions. In: Silkman RH (ed) Measuring efficiency: an assessment of data envelopment analysis, vol 32. Jossey-Bass, San Francisco, pp 73-105

Soltanifar M, Hosseinzadeh Lotfi F (2011) The voting analytic hierarchy process method for discriminating among efficient decision making units in data envelopment analysis. Comput Ind Eng 60:585-592

Tavana M, LoPinto F, Smither JW (2007) A hybrid distance-based ideal-seeking consensus ranking model. J Appl Math Decis Sci 11:1-18

Tavana M, LoPinto F, Smither JW (2008) Examination of the similarity between a new sigmoid function-based consensus ranking method and four commonly-used algorithms. Int J Oper Res 3(4):384-398

Thompson RG Jr, Singleton FD, Trall RM, Smith BA (1986) Comparative site evaluations for locating a high-energy physics lab in Texas. Interfaces 16(6):35-49

Tohidi G, Razavyan S (2012) An L1-norm method for generating all of efficient solutions of multi-objective integer linear programming problem. J Ind Eng Int 8(1):17

Wang YM, Chin KS (2007) Discriminating DEA efficient candidates by considering their least relative total scores. J Comput Appl Math 206:209-215

Wang NS, Yia RH, Liu D (2008) A solution method to the problem proposed by Wang in voting systems. J Comput Appl Math 221:106-113

Wu J, Dai L, Chiclana F, Fujita H, Herrera-Viedma E (2018) A minimum adjustment cost feedback mechanism-based consensus model for group decision making under social network with distributed linguistic trust. Inf Fusion 41:232-242

Zerafat Angiz L, Emrouznejad M, Mustafa A, Rashidi Komijan A (2009) Selecting the most preferable alternatives in a group decision making problem using DEA. Expert Syst Appl 36:9599-9602

Zhang N, Gong ZW, Chiclana F (2017) Minimum cost consensus models based on random opinions. Expert Syst Appl 89:149-159

Ziari S (2016) An alternative transformation in ranking using 11-norm in data envelopment analysis. J Ind Eng Int 12(3):401-405

Ziari S, Raissi S (2016) Ranking efficient DMUs using minimizing distance in DEA. J Ind Eng Int 12(2):237-242 